TRANSACTIONS OF THE

AMERICAN MATHEMATICAL SOCIETY

Volume 359, Number 1, January 2007, Pages 333-348

S 0002-9947(06)04058-X

Article electronically published on August 15, 2006

\title{
THE LEFSCHETZ PROPERTY, FORMALITY AND BLOWING UP IN SYMPLECTIC GEOMETRY
}

\author{
GIL RAMOS CAVALCANTI
}

\begin{abstract}
In this paper we study the behaviour of the Lefschetz property under the blow-up construction. We show that it is possible to reduce the dimension of the kernel of the Lefschetz map if we blow up along a suitable submanifold satisfying the Lefschetz property. We use this, together with results about Massey products, to construct compact nonformal symplectic manifolds satisfying the Lefschetz property.
\end{abstract}

\section{INTRODUCTION}

In 15] Koszul introduced an operator $\delta$ for Poisson manifolds which consists of the exterior derivative twisted by the Poisson bivector. This operator was further studied by Brylinski 4, who pointed out some similarities between $\delta$ and $d^{*}$, the Riemannian adjoint of $d$. He called forms that are both $d$ and $\delta$ closed symplectic harmonic. Later, Yan [26] and Mathieu [17] proved independently that the existence of a harmonic representative in each cohomology class is equivalent to the strong Lefschetz property (or just Lefschetz property, for short).

Lefschetz property. A symplectic manifold $\left(M^{2 n}, \omega\right)$ satisfies the Lefschetz property at level $k$ if the map

$$
\left[\omega^{n-k}\right]: H^{k}(M) \rightarrow H^{2 n-k}(M)
$$

is surjective. It satisfies the Lefschetz property if these maps are surjective for $0 \leq k \leq n$.

Recently, Merkulov [20] proved that, in a compact symplectic manifold, the existence of symplectic harmonic forms on each cohomology class (and therefore the Lefschetz property) is equivalent to the following $d \delta$-lemma.

$d \delta$-lemma. A symplectic manifold satisfies the $d \delta$-lemma if

$$
\operatorname{Im} d \cap \operatorname{ker} \delta=\operatorname{Im} \delta \cap \operatorname{ker} d=\operatorname{Im} d \delta .
$$

Therefore we have the following implications for a compact symplectic manifold:

$$
\text { Lefschetz property } \Leftrightarrow \text { Harmonic representatives } \Leftrightarrow d \delta \text {-lemma. }
$$

Although originally thought of as an analogue of $d^{*}$, the existence of a $d \delta$-lemma draws similarities between $\delta$ and the complex $d^{c}=-i(\partial-\bar{\partial})$. More evidence of

Received by the editors November 14, 2004.

2000 Mathematics Subject Classification. Primary 53D35; Secondary 57R19.

Key words and phrases. Strong Lefschetz property, symplectic blow-up, Massey products.

This research was supported by CAPES (Coordenação de Aperfeiçoamento de Pessoal de Nível Superior, Ministério da Educação e Cultura), Brazilian Government, Grant 1326/99-6.

(C)2006 American Mathematical Society 333 
the connection between these operators comes from generalized complex geometry, a theory recently introduced by Hitchin [13] and further developed by Gualtieri 12. As pointed out by Gualtieri, both $d^{c}$ and $\delta$ are particular cases of a general operator $d^{\mathcal{J}}$ on a generalized complex manifold, and implications of the $d d^{\mathcal{J}}$-lemma have been studied in the author's thesis [5].

In this paper we investigate whether two particular properties of the standard $d d^{c}$-lemma can be translated to the symplectic case. First, it is a result of Parshin 23 that the $d d^{c}$-lemma is preserved by holomorphic rational equivalences, in particular, by the blow-up along a complex submanifold. Secondly, it implies that the manifold is formal in the sense of Sullivan [7].

As far as the first point is concerned, we study how the Lefschetz property, and hence the $d \delta$-lemma, behaves under the operation of symplectic blow-up introduced by McDuff [18. McDuff used symplectic blow-up to give the first simply-connected example of a non-Kähler symplectic manifold, the blow-up of $\mathbb{C} P^{5}$ along a symplectically embedded Thurston manifold (the nilmanifold with structure $(0,0,0,12)$ ). This example of McDuff fails to be Kähler, since it does not satisfy the Lefschetz property. This means that even if the ambient space satisfies the $d \delta$-lemma, the blown-up manifold may not do the same.

In this paper we study systematically how the Lefschetz property behaves under the blow-up, in particular we seek conditions under which we can assure that the blown-up manifold will satisfy the Lefschetz property. We prove that this is the case if both submanifold and ambient manifold satisfy the property and the codimension is high enough (see Theorem 2.2). Moreover we study the blow-down map and show that even if the blown-up manifold satisfies the Lefschetz property, the original ambient manifold will not necessarily do so (see Theorem 2.1 and Proposition 2.2). Together with McDuff's example, this shows that we cannot decide whether the blow-up will or will not satisfy the Lefschetz property based solely on the ambient manifold.

The second purpose of this paper is to answer the question of correlation of the $d \delta$-lemma and formality. One of the implications is known to be false: Gompf produced a simply-connected 6-manifold which does not satisfy the Lefschetz property [10, but which is formal by Miller's result 21]. The converse implication had been conjectured by Babenko and Taimanov [2] and was the object of study of other papers [14, 16].

Our starting point in this case is that Babenko and Taimanov studied thoroughly the behaviour of Massey products under blow-up 2, 1, and these tend to 'survive' in the blow-up, which is markedly different from the behaviour of the Lefschetz property. Using this approach, we produce an example of a compact nonformal symplectic manifold satisfying the Lefschetz property by blowing up a 6-nilmanifold along a suitable torus. We also produce a 4-dimensional example using Donaldson submanifolds 8 and results of Fernández and Muñoz [9, and, with a further blowup, we obtain a simply-connected 12-dimensional nonformal compact symplectic manifold satisfying the Lefschetz property.

This paper is organized as follows. In the first section we explain briefly how the blow-up is done in symplectic geometry and derive the cohomology algebra of the blow-up from the cohomologies of the ambient manifold and submanifold as well as the Chern and Thom classes of the normal bundle of the submanifold. In Section 2. we study how the Lefschetz property behaves under blow-up, initially in the case 
of the blow-up along an embedded surface and later the general case. In Section 3 we recall the definition of Massey products, their relation with formality and their behaviour under blow-up. In Section 4 we provide examples of compact nonformal symplectic manifolds satisfying the Lefschetz property.

The author has been informed that other examples of compact nonformal symplectic manifolds satisfying the Lefschetz property have been found independently by Amorós and Kotschick using different methods.

\section{The SYMPLECTIC BLOW-UP}

We begin by giving a description of the cohomology ring of the blown-up manifold in terms of the cohomology rings of the ambient manifold and the embedded submanifold, and the Chern and Thom classes of the normal bundle of the embedding. We shall outline the blow-up construction in order to fix some notation. For a detailed presentation we refer to [18].

Assume that $i:\left(M^{2 d}, \sigma\right) \hookrightarrow\left(X^{2 n}, \omega\right)$ is a symplectic embedding, with $M$ compact. Let $k=n-d$. Under these circumstances we can choose a complex structure in $T X$ that restricts to one in $T M$, and hence also to the normal bundle $E \stackrel{\pi}{\rightarrow} M$. Therefore $E$ is a complex bundle over $M$, and one can form its projectivization

$$
\mathbb{C} P^{k-1} \longrightarrow \tilde{M} \longrightarrow M
$$

and also form the "tautological" line bundle $\tilde{E}$ over $\tilde{M}$ : the subbundle of $\tilde{M} \times E$ whose fibers are the elements $\{([v], \lambda v), \lambda \in \mathbb{C}\}$. We have the following commutative diagram:

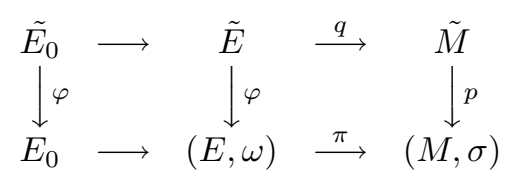

where $q$ and $\varphi$ are the projections over $\tilde{M}$ and $E$ respectively, $E_{0}$ is the complement of the zero section in $E$, and $\tilde{E}_{0}$ the complement of the zero section in $\tilde{E}$.

It is easily seen, $E_{0}$ and $\tilde{E}_{0}$ are diffeomorphic via $\varphi$. Furthermore, if we let $V$ be a sufficiently small disc subbundle in $E$ with its canonical symplectic structure $\omega$, then it is symplectomorphic to a neighbourhood of $M \subset X$ and we identify the two from now on. Letting $\tilde{V}=\varphi^{-1}(V)$, we can form the manifold

$$
\tilde{X}=\overline{X-V} \cup_{\partial V} \tilde{V} \text {. }
$$

Then, the map $\varphi$ can be extended to a map $f: \tilde{X} \rightarrow X$, being the identity in the complement of $\tilde{V}$. The manifold $\tilde{X}$ is the blow-up of $X$ along $M$, and $f: \tilde{X} \rightarrow X$ is the projection of the blow-up or the blow-down map.

Lemma 1.1 (McDuff [18]). There is a unique class $a \in H^{2}(\tilde{M})$ which restricts to the standard Kähler class on each fiber of $\tilde{M} \rightarrow M$ and pulls back to the trivial class in $\tilde{E}_{0}$. Moreover, $H^{\bullet}(\tilde{E}) \cong H^{\bullet}(\tilde{M})$ is a free module over $H^{\bullet}(M)$ with generators $1, a, \cdots, a^{k-1}$.

Theorem 1.1 (McDuff [18]). If the codimension of $M$ is at least 4, the fundamental groups of $X$ and the blown-up manifold $\tilde{X}$ are isomorphic. Further, there is a short exact sequence

$$
0 \rightarrow H^{*}(X) \rightarrow H^{*}(\tilde{X}) \rightarrow A^{*} \rightarrow 0
$$


where $A^{*}$ is the free module over $H^{*}(M)$ with generators $a, \cdots, a^{k-1}$. Moreover, there is a representative $\alpha$ of a with support in the tubular neighbourhood $V$ such that, for $\varepsilon$ small enough, the form $\tilde{\omega}=f^{*}(\omega)+\varepsilon \alpha$ is a symplectic form in $\tilde{X}$.

Remark. The symplectic structure in the blow-up, $\tilde{X}$, is not determined by the one in $X$. The presence of the parameter $\varepsilon$ in the symplectic form is just one indicator that this is the case. Furthermore, for each $\varepsilon$, the symplectic structure in $\tilde{X}$ also depends on other choices made during the construction, such as the almost complex structure taming $\omega$ and the identification of the normal bundle with the tubular neighbourhood. See [19], page 231.

Remark. If the submanifold has codimension less than 4, then the blow-up will be just $X$ again, and hence the theorem is trivially true.

Remark. As is observed by McDuff [18, the Leray-Hirsch theorem implies that $a^{k}$ is related to $a, \cdots, a^{k-1}$ in $\tilde{E}$ by

$$
a^{k}=-c_{k}-c_{k-1} a+\cdots-c_{1} a^{k-1},
$$

where the $c_{j}$ 's are the Chern classes of the normal bundle $E$.

In 24] it is shown that in $\tilde{X}$ this relation becomes

$$
a^{k}=-f^{*}(t)-c_{k-1} a+\cdots-c_{1} a^{k-1}
$$

where $t$ is the Thom class of the embedding $M \hookrightarrow X$ and $f: \tilde{X} \rightarrow X$ the projection of the blow-up.

With this, we have a complete description of the cohomology ring of $\tilde{X}$.

$$
H^{i}(\tilde{X})=H^{i}(X) \oplus H^{i-2}(M) a \oplus \cdots \oplus H^{i-2 k+2}(M) a^{k-1}
$$

and for $v_{1}, v_{2} \in H^{*}(X)$ and $u_{1}, u_{2} \in H^{*}(M)$,

$$
\begin{cases}f^{*}\left(v_{1}\right) \wedge f^{*}\left(v_{2}\right) & =f^{*}\left(v_{1} \wedge v_{2}\right), \\ f^{*}\left(v_{1}\right) a & =i^{*}\left(v_{1}\right) a, \\ u_{1} a \wedge u_{2} a & =u_{1} \wedge u_{2} a^{2}, \\ a^{k} & =-f^{*}(t)-c_{k-1} a+\cdots-c_{1} a^{k-1}, \\ f^{*}(t) \wedge u_{1} & =f^{*}\left(t \wedge u_{1}\right), \text { the Thom map extended to } X .\end{cases}
$$

\section{The Lefschetz PROPERTy AND BLOWING UP}

Now we move on to study how the Lefschetz property behaves under blow-up. The first case to look at would be the blow-up of a point, but, as we will see, this does not change the kernel of the Lefschetz map at any level (cf. Theorem 2.1). The next case would be a surface. Here, on the one hand, the situation is simple enough for us to be able to give a fairly complete account of what happens, and, on the other, we can already see that in this case it is possible to decrease the dimension of the kernel of the Lefschetz map. 
2.1. Blowing up along a surface. Assume that $i:\left(M^{2}, \sigma\right) \hookrightarrow\left(X^{2 n}, \omega\right)$ is a surface symplectically embedded in $X, M$ and $X$ are compact, and let $\tilde{X}$ be the blow-up of $X$ along $M$. In $H^{1}(\tilde{X})=f^{*}\left(H^{1}(X)\right)$ things go as follows:

$$
\begin{aligned}
\left(f^{*}(\omega)+\varepsilon a\right)^{n-1} f^{*}(v) & =f^{*}\left(\omega^{n-1} v\right)+\varepsilon^{n-1} a^{n-1} i^{*} v \\
& =f^{*}\left(\omega^{n-1} v-\varepsilon^{n-1} t v\right),
\end{aligned}
$$

and if Lefschetz holds for $X$ and $\varepsilon$ is small enough, Lefschetz will also hold for $\tilde{X}$, or, more generally, $\operatorname{dim}\left(\operatorname{ker}\left(\tilde{\omega}^{n-1}\right)\right) \leq \operatorname{dim}\left(\operatorname{ker}\left(\omega^{n-1}\right)\right)$. Now we proceed to show that in certain conditions the inequality holds.

Lemma 2.1. Let $i:\left(M^{2}, \sigma\right) \hookrightarrow\left(X^{2 n}, \omega\right)$ be a symplectic embedding, let $M$ and $X$ be compact and let $t$ be the Thom class of this embedding. The following are equivalent:

(1) There are $v_{1}, v_{2} \in H^{1}(X)$ in $\operatorname{ker}\left(\omega^{n-1}\right)$ such that $i^{*}\left(v_{1} \wedge v_{2}\right) \neq 0$.

(2) There exists $v_{1} \in \operatorname{ker}\left(\omega^{n-1}\right)$ such that $t \wedge v_{1} \notin \operatorname{Im}\left(\omega^{n-1}\right)$.

Proof. Assuming (1), by the defining property of the Thom class,

$$
\int_{X} t \wedge v_{1} \wedge v_{2}=\int_{M} i^{*}\left(v_{1} \wedge v_{2}\right) \neq 0
$$

and, since both $v_{1}$ and $v_{2}$ pair trivially with $\operatorname{Im}\left(\omega^{n-1}\right)$, but pair nontrivially with $t \wedge v_{i}$, we see that $t \wedge v_{i} \notin \operatorname{Im}\left(\omega^{n-1}\right)$. So (1) implies (2).

On the other hand, assume that there is a $v_{1}$ satisfying (2). Let $\left\{a_{i}\right\}$ be a basis for $\operatorname{ker}\left(\omega^{n-1}\right)$ and let $\left\{\tilde{a}_{i}\right\}$ be a basis for a complement. Since $\left(H^{1}(X)\right)^{*} \cong H^{2 n-1}(X)$, we can view the dual basis $\left\{a_{i}^{*}, \tilde{a}_{i}^{*}\right\}$ as a basis for $H^{2 n-1}(X)$. Then we note that $\operatorname{Im}\left(\omega^{n-1}\right) \subset \operatorname{span}\left\{\tilde{a}_{i}^{*}\right\}$, and since these spaces have the same dimension, they are the same. Therefore, the condition $t \wedge v_{1} \notin \operatorname{Im}\left(\omega^{n-1}\right)$ implies that it pairs nontrivially with some of the $a_{i}$. Let $v_{2}$ be such an $a_{i}$. Then again by the defining property of the Thom class we have

$$
\int_{M} i^{*}\left(v_{1} \wedge v_{2}\right)=\int_{X} t \wedge v_{1} \wedge v_{2} \neq 0
$$

and $i^{*}\left(v_{1} \wedge v_{2}\right) \neq 0$.

Lemma 2.2. If the equivalent conditions (1) and (2) of the previous lemma are satisfied and $\varepsilon$ is small enough, then

$$
\begin{aligned}
& \operatorname{dim}\left(\operatorname{ker}\left(\tilde{\omega}^{n-1}: H^{1}(\tilde{X}) \rightarrow H^{2 n-1}(\tilde{X})\right)\right) \\
& \quad \leq \operatorname{dim}\left(\operatorname{ker}\left(\omega^{n-1}: H^{1}(X) \rightarrow H^{2 n-1}(X)\right)\right)-2 .
\end{aligned}
$$

Proof. Let $V$ be a complement of $\operatorname{ker}\left(\omega^{n-1}\right)$ in $H^{1}(X)$ and let $v_{1}$ and $v_{2}$ be the cohomology classes satisfying condition (1) of Lemma 2.1] Then, since neither $t \wedge v_{1}$ nor $t \wedge v_{2}$ is in $\operatorname{Im}\left(\omega^{n-1}\right)$, for $\varepsilon$ small enough, 2.1) shows that $f^{*}\left(t \wedge v_{i}\right) \notin$ $\operatorname{Im}\left(\left.\tilde{\omega}^{n-1}\right|_{V}\right)$, since $\left.\tilde{\omega}^{n-1}\right|_{V}$ is simply a perturbation of the injection $\left.\omega^{n-1}\right|_{V}$. On the other hand, $\tilde{\omega}^{n-1} f^{*}\left(v_{i}\right)=-\varepsilon^{n-1} f^{*}\left(t \wedge v_{i}\right)$, and therefore $f^{*}\left(t \wedge v_{i}\right)$ is in the image of $\tilde{\omega}^{n-1}$, so

$$
\operatorname{dim}\left(\operatorname{Im}\left(\tilde{\omega}^{n-1}\right)\right) \geq \operatorname{dim}\left(\operatorname{Im}\left(\omega^{n-1}\right)\right)+2,
$$

and the result follows. 
Now we move on to $H^{2}(\tilde{X})=f^{*}\left(H^{2}(X)\right) \oplus a H^{0}(M)$, where we have

$$
\begin{aligned}
& \left(f^{*}(\omega)+\varepsilon a\right)^{n-2}\left(f^{*}\left(v_{2}\right)+a v_{0}\right) \\
& \quad=f^{*}\left(\omega^{n-2} v_{2}-\varepsilon^{n-2} t v_{0}\right)+\varepsilon^{n-3} a^{n-2}\left((n-2) \sigma v_{0}+\varepsilon\left(i^{*} v_{2}-c_{1} v_{0}\right)\right),
\end{aligned}
$$

and then we observe that the map above is a perturbation of

$$
f^{*}\left(v_{2}\right)+a v_{0} \mapsto f^{*}\left(\omega^{n-2} v_{2}\right)+\varepsilon^{n-3} a^{n-2}(n-2) \sigma v_{0} .
$$

Therefore for $\varepsilon$ small enough, Lefschetz will hold for $\tilde{X}$ if it holds for $X$, or more generally $\operatorname{dim}\left(\operatorname{ker}\left(\tilde{\omega}^{n-2}\right)\right) \leq \operatorname{dim}\left(\operatorname{ker}\left(\omega^{n-2}\right)\right)$.

Again, we may have the inequality.

Lemma 2.3. Let $i:\left(M^{2}, \sigma\right) \hookrightarrow\left(X^{2 n} \omega\right)$ be a symplectic embedding, let $M$ and $X$ be compact and let $t$ be the Thom class of this embedding. The following are equivalent:

(1) There exists $v \in \operatorname{ker} \omega^{n-2}$ such that $i^{*} v \neq 0$.

(2) The Thom class $t$ is not in the image of $\omega^{n-2}$.

Proof. The proof is the same as the one for Lemma 2.1. Assuming (1), by definition of the Thom class,

$$
\int_{X} t v=\int_{M} i^{*} v \neq 0
$$

On the other hand any vector in the kernel of $\omega^{n-2}$ pairs trivially with $\operatorname{Im}\left(\omega^{n-2}\right)$, so $t \notin \operatorname{Im}\left(\omega^{n-2}\right)$.

Conversely, we again let $\left\{a_{i}\right\}$ be a basis for $\operatorname{ker}\left(\omega^{n-2}\right),\left\{\tilde{a}_{i}\right\}$ a basis for a complement and $\left\{a_{i}^{*}, \tilde{a}_{i}^{*}\right\}$ the dual basis, and again identify the dual space with $H^{2 n-2}(X)$. Then we see that $\operatorname{Im}\left(\omega^{n-2}\right)=\operatorname{span}\left\{\tilde{a}_{i}^{*}\right\}$ and, since $t \notin \operatorname{Im}\left(\omega^{n-2}\right), t$ must pair nontrivially with at least one of the $a_{i}$ 's. Call it $v$.

Lemma 2.4. If the equivalent conditions (1) and (2) of the previous lemma are satisfied and $\varepsilon$ is small enough, then

$$
\begin{aligned}
& \operatorname{dim}\left(\operatorname{ker}\left(\tilde{\omega}^{n-2}: H^{2}(\tilde{X}) \rightarrow H^{2 n-2}(\tilde{X})\right)\right) \\
& \quad=\operatorname{dim}\left(\operatorname{ker}\left(\omega^{n-2}: H^{2}(X) \rightarrow H^{2 n-2}(X)\right)\right)-1 .
\end{aligned}
$$

Proof. By conveniently choosing $v_{2}$ and $v_{0}$ in (2.2),

$$
\begin{aligned}
\left(f^{*}(\omega)\right. & +\varepsilon a)^{n-2}\left(f^{*}\left(v_{2}\right)+a v_{0}\right) \\
& =\underbrace{f^{*}\left(\omega^{n-2} v_{2}-\varepsilon^{n-2} t v_{0}\right)}_{H^{2 n-2}(X)}+\varepsilon^{n-3} a^{n-2}\left((n-2) \sigma v_{0}+\varepsilon(\underbrace{i^{*} v_{2}}_{\neq 0}-c_{1} v_{0})\right)
\end{aligned}
$$

the term in $H^{2 n-2}(X)$ can be made equal to any pre-chosen element in $\operatorname{Im}\left(\omega^{n-2}\right) \oplus$ $\operatorname{span}\{t\}$. Once $v_{2}$ and $v_{0}$ are chosen, changing $v_{2}$ by an element in $\operatorname{ker}\left(\omega^{n-2}\right)$ does not affect the result. On the other hand, by varying $v_{2}$ by an element in $\operatorname{ker}\left(\omega^{n-2}\right)$ the coefficient of $a$ can be made equal to anything in $H^{2}(M)$. Therefore $\operatorname{dim}\left(\operatorname{Im}\left(\tilde{\omega}^{n-2}\right)\right)=\operatorname{dim}\left(\operatorname{Im}\left(\omega^{n-2}\right)\right)+2$ and $\operatorname{dim}\left(H^{2}(\tilde{X})\right)=\operatorname{dim}\left(H^{2}(X)\right)+1$, hence the result follows.

Finally, we finish the study of the blow-up along surfaces claiming that, for $i>2$,

$$
\operatorname{dim}\left(\operatorname{ker}\left(\tilde{\omega}^{n-i}\right)\right)=\operatorname{dim}\left(\operatorname{ker}\left(\omega^{n-i}\right)\right) .
$$


Indeed, if $v_{i} \in \operatorname{ker}\left(\omega^{n-i}\right)$, then $i^{*}\left(v_{i}\right)=0$, since it has degree greater than 2, and therefore $a f^{*}\left(v_{i}\right)=a i^{*}\left(v_{i}\right)=0$ and

$$
\left(f^{*}(\omega)+\varepsilon a\right)^{n-i} f^{*}\left(v_{i}\right)=f^{*}\left(\omega^{n-i} v_{i}\right)=0,
$$

so $f^{*}\left(\operatorname{ker}\left(\omega^{n-i}\right)\right) \subset \operatorname{ker}\left(\tilde{\omega}^{n-i}\right)$.

Conversely, assuming $i$ even (the odd case is analogous),

$$
\begin{aligned}
\left(f^{*}(\omega)+\varepsilon a\right)^{n-i} & \left(f^{*}\left(v_{i}\right)+a^{\frac{i}{2}} v_{0}+a^{\frac{i-2}{2}} v_{2}\right) \\
& =f^{*}\left(\omega^{n-i} v_{i}\right)+\varepsilon^{n-i-1} a^{n-\frac{i}{2}-1}\left((n-i) \sigma v_{0}+\varepsilon v_{2}\right)+\varepsilon^{n-i} a^{n-\frac{i}{2}} v_{0},
\end{aligned}
$$

and therefore $f^{*}\left(v_{i}\right)+a^{\frac{i}{2}} v_{0}+a^{\frac{i-2}{2}} v_{2}$ will be in $\operatorname{ker}\left(\tilde{\omega}^{n-i}\right)$ if, and only if, $v_{0}=0$ (by the coefficient of $\left.a^{n-\frac{i}{2}}\right), v_{1}=0$ (by the coefficient of $a^{n-\frac{i}{2}-1}$ ) and $v_{i} \in \operatorname{ker}\left(\omega^{n-i}\right)$, establishing the reverse inclusion.

So we have proved:

Theorem 2.1. Let $i: M^{2} \hookrightarrow X^{2 n}$ be a symplectic embedding, let $M$ and $X$ be compact and let $\tilde{X}$ be the blow up of $X$ along $M$. Then, for $\varepsilon$ small enough,

- for $i>2$,

$$
\operatorname{dim}\left(\operatorname{ker}\left(\tilde{\omega}^{n-i}\right)\right)=\operatorname{dim}\left(\operatorname{ker}\left(\omega^{n-i}\right)\right),
$$

in particular, Lefschetz holds at level $i$ in $\tilde{X}$ if, and only if, it does so in $X$;

- if there is an element in $\operatorname{ker}\left(\omega^{n-2}\right)$ that restricts to a nonzero element in $H^{2}(M)$, then

$$
\operatorname{dim}\left(\operatorname{ker}\left(\tilde{\omega}^{n-2}\right)\right)=\operatorname{dim}\left(\operatorname{ker}\left(\omega^{n-2}\right)\right)-1,
$$

otherwise these kernels have the same dimension;

- if there are elements $v_{1}, v_{2} \in \operatorname{ker}\left(\omega^{n-1}\right)$ such that $i^{*}\left(v_{1} \wedge v_{2}\right) \neq 0$, then

$$
\operatorname{dim}\left(\operatorname{ker}\left(\tilde{\omega}^{n-1}\right)\right) \leq \operatorname{dim}\left(\operatorname{ker}\left(\omega^{n-1}\right)\right)-2,
$$

otherwise

$$
\operatorname{dim}\left(\operatorname{ker}\left(\tilde{\omega}^{n-1}\right)\right) \leq \operatorname{dim}\left(\operatorname{ker}\left(\omega^{n-1}\right)\right) .
$$

2.2. The general case. Now we treat the general case of the blow-up. Our main objective is to prove that if both $M^{2 d}$ and $X^{2 n}$ satisfy the Lefschetz property, so does the blow-up of $X$ along $M$, although in the course of this proof we obtain slightly more, including a generalization of Lemma 2.4 The first part of the proof was already encountered at the end of the 2-dimensional case.

Proposition 2.1. Assume that $\left(M^{2 d}, \sigma\right) \hookrightarrow\left(X^{2 n}, \omega\right)$ is a symplectic embedding with $M$ and $X$ compact and $2 d<n$. Let $\tilde{X}$ be the blown-up manifold. Then, for $i>2 d$

$$
\operatorname{dim}\left(\operatorname{ker}\left(\tilde{\omega}^{n-i}\right)\right)=\operatorname{dim}\left(\operatorname{ker}\left(\omega^{n-i}\right)\right) .
$$

In particular, $\tilde{X}$ will satisfy the Lefschetz property at level $i>2 d$ if, and only if, $X$ does.

Remark. The condition $2 d<n$ is there only so that we can talk about a Lefschetz map at level $i>2 d$, and this proposition says that we cannot change the dimension of the kernel of the Lefschetz map beyond the dimension of the submanifold along which we are blowing-up. 
Proof. First we return to our usual notation and let $k=n-d$. Let $v_{i} \in \operatorname{ker}\left(\omega^{n-i}\right) \subset$ $H^{i}(X)$ and consider the cohomology class $f^{*}\left(v_{i}\right) \in H^{i}(\tilde{X})$. The restriction of $v_{i}$ to $M$ is zero, since the degree of $v_{i}$ is greater than the dimension of $M$. Therefore $a v_{i}=0$ and

$$
\left(f^{*}(\omega)+\varepsilon a\right)^{n-i} f^{*}\left(v_{i}\right)=f^{*}\left(\omega^{n-i} v_{i}\right)=0 .
$$

Thus, $f^{*}\left(\operatorname{ker}(\omega)^{n-i}\right) \subset \operatorname{ker}\left(\tilde{\omega}^{n-i}\right)$.

On the other hand assume that $v=f^{*}\left(v_{i}\right)+a v_{i-2}+\cdots+a^{l} v_{i-2 l}$ is an element of the kernel of $\tilde{\omega}^{n-i}$. We may further assume that the last term above, $v_{i-2 l}$, is not zero or else $v$ is of the form $f^{*}\left(v_{i}\right)$. From $v \in \operatorname{ker}(\tilde{\omega})$ we have

$$
\begin{aligned}
0 & =\left(f^{*}(\omega)+\varepsilon a\right)^{n-i}\left(f^{*}\left(v_{i}\right)+a v_{i-2}+\cdots+a^{l} v_{i-2 l}\right) \\
& =f^{*}\left(\omega^{n-i} v_{i}\right)+\sum_{j=0, m=1}^{n-i, l} \varepsilon^{j}\left(\begin{array}{c}
n-i \\
j
\end{array}\right) a^{j+m} \sigma^{n-i-j} v_{i-2 m} .
\end{aligned}
$$

Since $i>2(n-k)$, the degree of the element above is $2 n-i<2 k$ and therefore the highest power of $a$ in the expression above is still smaller than $k$. Hence the coefficient of $a^{l+n-i}$, which is $v_{i-2 l}$, must vanish. Thus we had $v=f^{*}\left(v_{i}\right)$ from the beginning, and the expression above reduces to

$$
0=\left(f^{*}(\omega)+\varepsilon a\right)^{n-i}\left(f^{*}\left(v_{i}\right)\right)=f^{*}\left(\omega^{n-i} v_{i}\right)
$$

and $v \in f^{*}\left(\operatorname{ker}\left(\omega^{n-i}\right)\right)$, which shows the reverse inclusion and proves the proposition.

Proposition 2.2. Assume that $i:\left(M^{2 d}, \sigma\right) \hookrightarrow\left(X^{2 n}, \omega\right)$ is a symplectic embedding with $M$ and $X$ compact and $2 d<n$. Let $\tilde{X}$ be the blown-up manifold. If there is a $v \in \operatorname{ker}\left(\omega^{n-2 d}\right)$ such that $i^{*} v \neq 0$, then

$$
\operatorname{dim}\left(\operatorname{ker}\left(\tilde{\omega}^{n-2 d}\right)\right)=\operatorname{dim}\left(\operatorname{ker}\left(\omega^{n-2 d}\right)\right)-1 ;
$$

otherwise these kernels have the same dimension, as long as $\varepsilon$ is small enough. In particular, if $X$ has the Lefschetz property at level $2 d$, so does $\tilde{X}$.

Proof. Initially we observe that the same argument used in Lemma 2.3 shows that the existence of $v \in \operatorname{ker}\left(\omega^{n-2 d}\right)$ such that $i^{*} v \neq 0$ is equivalent to the fact that the Thom class, $t$, of the embedding is not in the image of $\omega^{n-2 d}$. Now we let $k=n-d$ and write down the Lefschetz map at level $2 d$

$$
\begin{aligned}
\left(f^{*}(\omega)\right. & +\varepsilon a)^{n-2 d}\left(f^{*}\left(v_{2 d}\right)+a v_{2 d-2}+\cdots+a^{d} v_{0}\right) \\
= & f^{*}\left(\omega^{n-2 d} v_{2 d}-\varepsilon^{n-2 d} v_{0} t\right) \\
& +\sum_{i=k-d}^{k-1} a^{i}\left(\left(\sum_{l \geq i-n+2 d}^{d}\left(\begin{array}{c}
n-2 d \\
i-l
\end{array}\right) \varepsilon^{i-l} \sigma^{n-2 d-i+l} v_{2(d-l)}\right)-\varepsilon^{n-2 d} v_{0} c_{k-i}\right)
\end{aligned}
$$

where the $c_{i}$ 's are the Chern classes of the normal bundle of $M$. Then we claim that we can make it equal to any element in

$$
f^{*}\left(\operatorname{Im}\left(\omega^{n-2 d}\right) \oplus \operatorname{span}\{t\}\right) \oplus a^{k-d} H^{2 d}(M) \oplus \cdots \oplus a^{k-1} H^{2}(M) .
$$

The idea is the following: the system above is triangular and therefore easy to solve. Indeed, let $f^{*}\left(w_{2(n-d)}\right)+a^{k-d} w_{2 d}+\cdots+a^{k-1} w_{2}$ be an element of the space $(*)$. We start by choosing $v_{2 d}$ and $v_{0}$ so that $\omega^{n-2 d} v_{2 d}-\varepsilon^{n-2 d} t v_{0}$ equals $w_{2(n-d)}$. 
Observe that we can still change $v_{2 d}$ by any element in the kernel of $\omega^{n-2 d}$. Now look at the coefficient of $a^{k-1}$ in (2.3):

$$
\varepsilon^{n-2 d} v_{2}+(n-2 d) \varepsilon^{n-2 d-1} \sigma v_{0}-\varepsilon^{n-2 d} v_{0} c_{1}:=\varepsilon^{n-2 d} v_{2}+F\left(v_{0}\right) .
$$

Since we have already chosen $v_{0}$, we can now choose $v_{2}$ so that the expression above equals $w_{2}$.

Assuming by induction that $v_{2 j}$ have already been chosen for $j<j_{0}<k-d$ so that the coefficient of $a^{k-j}$ is $w_{2 j}$, we see that the coefficient of $a^{k-j_{0}}$ in (2.3) is of the form

$$
\varepsilon^{n-2 d} v_{2 j_{0}}+F\left(v_{0}, \cdots, v_{2 j_{0}-2}\right)
$$

where $F$ is a function. Then again we can choose $v_{2 j_{0}}$ so as to have the desired equality.

Finally the coefficient of $a^{k-d}$ is of the form

$$
\varepsilon^{n-2 d} i^{*} v_{2 d}+F\left(v_{0}, \cdots, v_{2 d-2}\right) \in H^{2 d}(M) .
$$

Then, changing $v_{2 d}$ by a multiple of the element in $\operatorname{ker}\left(\omega^{n-2 d}\right)$ whose restriction to $M$ is nonvanishing, we can make this coefficient equal $w_{2 d}$.

Now a simple counting of the dimensions involved shows that

$$
\operatorname{dim}\left(\operatorname{ker}\left(\tilde{\omega}^{n-2 d}\right)\right)=\operatorname{dim}\left(\operatorname{ker}\left(\omega^{n-2 d}\right)\right)-1 .
$$

In order to prove the "otherwise" case, we start observing that if for every $v \in \operatorname{ker}\left(\omega^{n-2 d}\right), i^{*} v=0$, then $f^{*}(\operatorname{ker}(\omega)) \subset \operatorname{ker}\left(\tilde{\omega}^{n-2 d}\right)$. Therefore we immediately have $\operatorname{dim}\left(\operatorname{ker}\left(\omega^{n-2 d}\right)\right) \leq \operatorname{dim}\left(\operatorname{ker}\left(\tilde{\omega}^{n-2 d}\right)\right)$.

The reverse inequality is similar to what we have done so far and also to the subject of Proposition 2.3, so we shall omit its proof.

Before we can tackle the case $i<2 d$ we have to recall Yan [26].

Lemma 2.5. If $\left(M^{2 d}, \sigma\right)$ satisfies the Lefschetz property, there is a splitting of every cohomology class into primitive elements:

$$
H^{i}(M)=P_{i} \oplus \operatorname{Im}(\sigma)
$$

where $P_{i}$ is defined by

$$
P_{i}=\left\{v \in H^{i}(M) \mid \sigma^{d-i+1} v=0\right\},
$$

if $i \leq d$ and $P_{i}=\{0\}$ otherwise. The elements in $P_{i}$ are called primitive $i-$ cohomology classes.

Hence, we can write every $v \in H^{i}, i \leq j$, in a unique way as $v=v^{0}+v^{1} \sigma+\cdots+$ $v^{[i / 2]} \sigma^{[i / 2]}$, with $v^{j}$ primitive. Observe that if $i>d$, then the first few terms in this decomposition will vanish simply because $P_{j}=\{0\}$ for $j>d$. Again, the notation for the splitting above will be used consistently in the sequence.

Proposition 2.3. Let $i:\left(M^{2 d}, \sigma\right) \hookrightarrow\left(X^{2 n}, \omega\right)$ be a symplectic embedding with $M$ and $X$ compact and $2 d<n$. Assume further that $M$ satisfies the Lefschetz property. Then, for $\varepsilon$ small enough and $i \leq 2 d$,

$$
\operatorname{dim}\left(\operatorname{ker}\left(\tilde{\omega}^{n-i}\right)\right) \leq \operatorname{dim}\left(\operatorname{ker}\left(\omega^{n-i}\right)\right) .
$$

In particular, if $X$ satisfies the Lefschetz property at level $i$, so does $\tilde{X}$. 
Proof. First we observe that the cases of $i$ odd and $i$ even can be treated similarly, but for simplicity we shall work out only the even case: $2 i$.

We want to take the limit $\varepsilon \rightarrow 0$ in the map $\tilde{\omega}^{n-2 i}$, but, as it stands, the resulting map will clearly have a big kernel. So, what we shall do is to find linear maps $A_{\varepsilon}$ and $B_{\varepsilon}$ such that $\lim _{\varepsilon \rightarrow 0} B_{\varepsilon} \tilde{\omega}^{n-2 i} A_{\varepsilon}$ has kernel $f^{*}\left(\operatorname{ker}\left(\omega^{n-2 i}\right)\right)$. From this we shall conclude that the dimension of the kernel of $\tilde{\omega}^{n-2 i}$ is at most the dimension of the kernel of $\omega^{n-2 i}$ as long as $\varepsilon$ is small enough.

We define $A_{\varepsilon}: H^{2 i}(\tilde{X}) \rightarrow H^{2 i}(\tilde{X})$ by

$$
A_{\varepsilon}\left(f^{*}\left(v_{2 i}\right)+\sum_{j=0}^{i-1} a^{i-j} v_{2 j}\right)=f^{*}\left(v_{2 i}\right)+\sum_{j=0}^{i-1} \frac{1}{\varepsilon^{j}} a^{i-j} v_{2 j} .
$$

Also, $B_{\varepsilon}: H^{2 n-2 i}(\tilde{X}) \rightarrow H^{2 n-2 i}(\tilde{X})$ by

$$
\begin{aligned}
& B_{\varepsilon}\left(f^{*}\left(v_{2 n-2 i}\right)+\sum_{j=0}^{i-1} a^{n-d-i+j} v_{2 d-2 j}\right) \\
& =f^{*}\left(v_{2 n-2 i}\right)+\sum_{j=0}^{i-1} \frac{1}{\varepsilon^{n-d-2 i+j}} a^{n-d-i+j} v_{2 d-2 j} .
\end{aligned}
$$

Now we move on to write the map $\lim _{\varepsilon \rightarrow 0} B_{\varepsilon} \tilde{\omega}^{n-2 i} A_{\varepsilon}$ :

$$
\lim _{\varepsilon \rightarrow 0} B_{\varepsilon} \tilde{\omega}^{n-2 i} A_{\varepsilon}=f^{*}\left(\omega^{n-2 i} v_{2 i}\right)+\sum_{j=0}^{i-1} a^{n-d-i+j} \sum_{l=0}^{i-1} b_{d-j-l} \sigma^{d-j-l} v_{2 l},
$$

where $b_{j}=\left(\begin{array}{c}n-2 i \\ j\end{array}\right)$ are the binomial coefficients.

We can further split the cohomology classes $v_{2 l}$ into their primitive parts, according to Lemma 2.5. $v_{2 l}=v_{2 l}^{0}+\sigma v_{2 l}^{1}+\cdots+\sigma^{l} v_{2 l}^{l}$. With that, elements of $H^{2 i}(\tilde{X})$ will be in the kernel of the map above only if the coefficients of $a^{j} \sigma^{l}$ vanish. The only terms that will give us information about primitives of degree $2 l$ are the coefficients of $a^{k-i+l} \sigma^{d-2 l}, a^{k-i+l+1} \sigma^{d-2 l-1}, \ldots, a^{k-1} \sigma^{d-l-i+1}$, and the vanishing of these is equivalent to the following:

$$
\left(\begin{array}{ccccc}
b_{d-2 l} & b_{d-2 l-1} & \cdots & b_{d-l-i+2} & b_{d-l-i+1} \\
b_{d-2 l-1} & b_{d-2 l-2} & \cdots & b_{d-l-i+1} & b_{d-l-i} \\
\vdots & & \ddots & & \vdots \\
b_{d-2 l-i+2} & b_{d-2 l-i+1} & \cdots & b_{d-2 i+4} & b_{d-2 i+3} \\
b_{d-2 l-i+1} & b_{d-2 l-i} & \cdots & b_{d-2 i+3} & b_{d-2 i+2}
\end{array}\right)\left(\begin{array}{c}
v_{2 l}^{0} \\
v_{2 l+2}^{1} \\
\vdots \\
v_{2 i-4-l}^{i-2-l} \\
v_{2 i-2}^{i-1-l}
\end{array}\right)=0
$$

in the case $2 i<d$, and a similar matrix for $2 i>d$. What is important here is that in both cases the matrix will be constant along its anti-diagonals (it is a Toeplitz matrix) and the top right entry is nonzero. Now, if we can prove that all the matrices above are invertible, we will conclude that $f^{*}\left(v_{2 i}\right)+\sum a^{i-j} v_{2 j}$ is in the kernel of $\lim B_{\varepsilon} \tilde{\omega}^{n-2 i} A_{\varepsilon}$ if and only if $v_{2 j}=0$ for all $j<i$ and $v_{2 i} \in \operatorname{ker}\left\{\omega^{n-2 i}\right\}$. So the next lemma finishes the theorem. 
Lemma 2.6. Let $b_{j}^{n}=\left(\begin{array}{c}n \\ j\end{array}\right), n, j \in \mathbb{N}$. Then for any $p \in \mathbb{N}$

if $b_{k}^{n} \neq 0$.

$$
\Delta_{k}^{n, p+1}=\operatorname{det}\left(\begin{array}{ccccc}
b_{k+p}^{n} & b_{k+p-1}^{n} & \cdots & b_{k+1}^{n} & b_{k}^{n} \\
b_{k+p-1}^{n} & b_{k+p-2}^{n} & \cdots & b_{k}^{n} & b_{k-1}^{n} \\
\vdots & & \ddots & & \vdots \\
b_{k+1}^{n} & b_{k}^{n} & \cdots & b_{k-p+2}^{n} & b_{k-p+1}^{n} \\
b_{k}^{n} & b_{k-1}^{n} & \cdots & b_{k-p+1}^{n} & b_{k-p}^{n}
\end{array}\right) \neq 0
$$

Proof. Initially we observe that $b_{k}^{n} \neq 0$ if and only if $n \geq k \geq 0$ and for $n=k$ the matrix above has zeros above the anti-diagonal and ones on it, so the determinant is a power of -1 . Further, by adding to each column the one to its right and using the binomial identity $b_{k}^{n}+b_{k-1}^{n}=b_{k}^{n+1}$ we get

$$
\Delta_{k}^{n, p+1}=\operatorname{det}\left(\begin{array}{ccccc}
b_{k+p}^{n+p} & b_{k+p-1}^{n+p-1} & \cdots & b_{k+1}^{n+1} & b_{k}^{n} \\
b_{k+p-1}^{n+p} & b_{k+p-2}^{n+p-1} & \cdots & b_{k}^{n+1} & b_{k-1}^{n} \\
\vdots & & \ddots & & \vdots \\
b_{k+1}^{n+p} & b_{k}^{n+p-1} & \cdots & b_{k-p+2}^{n+1} & b_{k-p+1}^{n} \\
b_{k}^{n+p} & b_{k-1}^{n+p-1} & \cdots & b_{k-p+1}^{n+1} & b_{k-p}^{n}
\end{array}\right) .
$$

Now it is easy to check that

$$
\Delta_{k}^{n+1, p+1}=\frac{(n+p+1) !(n-k) !}{n !(n+p-k+1) !} \Delta_{k}^{n, p+1},
$$

showing that $\Delta_{k}^{n+1, p+1}$ is nonzero if $\Delta_{k}^{n, p+1}$ is nonzero, and we obtain the result by induction.

These three propositions give us the following.

Theorem 2.2. Let $i:\left(M^{2 d}, \sigma\right) \hookrightarrow\left(X^{2 n}, \omega\right)$ be a symplectic embedding with $M$ and $X$ compact and both satisfying the Lefschetz property and $2 d<n$. Let $(\tilde{X}, \omega+\varepsilon \alpha)$ be the blow-up of $X$ along $M$ with the symplectic form from Theorem 1.1. Then, for $\varepsilon$ small enough, $\tilde{X}$ also satisfies the Lefschetz property.

\section{Massey products AND the BlOW-UP}

Having determined how the Lefschetz property behaves under blow-up, we turn our attention to formality. Here, we use Massey products to prove that manifolds are not formal, since formality implies that these products vanish. The object of this section is to prove that under mild codimension conditions, Massey products are preserved in the blow-up. This will allow us to find examples of nonformal symplectic manifolds in the next section.

The ingredients for a Massey product are $a_{12}, a_{23}, a_{34} \in \Omega^{\bullet}(M)$, three closed forms such that $a_{12} a_{23}$ and $a_{23} a_{34}$ are exact. Then, denoting $\bar{a}=(-1)^{|a|} a$, we define

$$
\begin{cases}\overline{a_{12}} a_{23} & =d a_{13}, \\ \overline{a_{23}} a_{34} & =d a_{24} .\end{cases}
$$

In this case, one can consider the element $\overline{a_{13}} a_{34}+\overline{a_{12}} a_{24}$. By the choice of $a_{13}$ and $a_{24}$ this form is closed, hence it represents a cohomology class. Observe, however, that $a_{13}$ and $a_{24}$ are not well defined and we can change them by any closed element. 
Hence the expression above does not define a unique cohomology class but instead an element in the quotient $H^{\bullet}(M) / \mathcal{I}\left(\left[a_{1}\right],\left[a_{3}\right]\right)$, where $\mathcal{I}$ denotes the ideal generated by its arguments.

Definition. The triple Massey product or just triple product $\left\langle\left[a_{12}\right],\left[a_{23}\right],\left[a_{34}\right]\right\rangle$ of the cohomology classes $\left[a_{12}\right],\left[a_{23}\right]$ and $\left[a_{34}\right]$ with $\left[a_{12}\right]\left[a_{23}\right]=\left[a_{23}\right]\left[a_{34}\right]=0$ is the coset

$$
\left\langle\left[a_{12}\right],\left[a_{23}\right],\left[a_{34}\right]\right\rangle=\left[\overline{a_{12}} a_{24}+\overline{a_{13}} a_{34}\right]+\left(\left[a_{12}\right],\left[a_{34}\right]\right) \in H^{\bullet}(\mathcal{A}) / \mathcal{I}\left(\left[a_{12}\right],\left[a_{34}\right]\right),
$$

where $a_{13}$ and $a_{24}$ are defined by $(*)$.

Theorem 3.1. Let $i: M^{2(n-k)} \hookrightarrow X^{2 n}$ be a symplectic embedding with $M$ compact and let $\tilde{X}$ be the blown-up manifold. Then:

- if $X$ has a nontrivial triple Massey product, so does $\tilde{X}$,

- (Babenko and Taimanov [2]) if $M$ has a nontrivial triple Massey product and $k>3$, so does $\tilde{X}$.

Proof. We start with the first claim and assume the Massey product $\left\langle v_{1}, v_{2}, v_{3}\right\rangle$ is nonzero in $X$. This means that there is $u$ representing such a product with $[u] \notin \mathcal{I}\left(\left[v_{1}\right],\left[v_{3}\right]\right)$, the ideal generated by $\left[v_{1}\right]$ and $\left[v_{3}\right]$ in $H^{*}(X)$. If we consider the product $\left\langle f^{*} v_{1}, f^{*} v_{2}, f^{*} v_{3}\right\rangle$, we see that $f^{*} u$ is a representative for it. The question then is whether $f^{*}[u]$ is in the ideal $\left(f^{*}\left[v_{1}\right], f^{*}\left[v_{3}\right]\right)$. Let us assume there was a relation of the kind

$f^{*}[u]=f^{*}\left[v_{1}\right]\left(f^{*} \xi_{1}+a \zeta_{1}^{1}+\cdots+a^{k-1} \zeta_{1}^{k-1}\right)+f^{*}\left[v_{3}\right]\left(f^{*} \xi_{3}+a \zeta_{3}^{1}+\cdots+a^{k-1} \zeta_{3}^{k-1}\right)$.

Then, using the product rules (1.2),

$f^{*}[u]=f^{*}\left(\left[v_{1}\right] \xi_{1}+\left[v_{3}\right] \xi_{3}\right)+a\left(i^{*}\left[v_{1}\right] \zeta_{1}^{1}+i^{*}\left[v_{3}\right] \zeta_{3}^{1}\right)+\cdots+a^{k-1}\left(i^{*}\left[v_{1}\right] \zeta_{1}^{k-1}+i^{*}\left[v_{3}\right] \zeta_{3}^{k-1}\right)$.

Now, since the sum above is a direct one, all the coefficients of the powers of $a$ must vanish and the following must hold:

$$
f^{*}[u]=f^{*}\left(\left[v_{1}\right] \xi_{1}+\left[v_{3}\right] \xi_{3}\right) .
$$

Since $f^{*}$ is an injection, we conclude that $[u] \in\left(\left[v_{1}\right],\left[v_{3}\right]\right)$, which contradicts our initial assumption.

Now we treat the second case. We start by assuming that $v_{1}, v_{2}$ and $v_{3} \in \Omega(M)$ are closed forms satisfying

$$
v_{1} \wedge v_{2}=d w_{1} \text { and } v_{2} \wedge v_{3}=d w_{2},
$$

with $\left[w_{1} v_{3}-(-1)^{\left|v_{1}\right|} v_{1} w_{2}\right] \notin\left(\left[v_{1}\right],\left[v_{3}\right]\right)$. Letting $\varphi: \tilde{V} \rightarrow V$ be the map of diagram (1.1) and $\pi: V \rightarrow M$ the projection of the disc bundle, we have the following relations in $H^{*}(\tilde{X})$ :

$$
\alpha \varphi^{*} \pi^{*} v_{1} \wedge \alpha \varphi^{*} \pi^{*} v_{2}=d\left(\alpha^{2} \varphi^{*} \pi^{*} w_{1}\right) \text { and } \alpha \varphi^{*} \pi^{*} v_{2} \wedge \alpha \varphi^{*} \pi^{*} v_{3}=d\left(\alpha^{2} \varphi^{*} \pi^{*} w_{2}\right) .
$$

The question then is again whether the cohomology class of the form

$$
\alpha \varphi^{*} \pi^{*} v_{1} \alpha^{2} \varphi^{*} \pi^{*} w_{1}-(-1)^{\left|v_{1}\right|} \alpha \varphi^{*} \pi^{*} v_{1} \alpha^{2} \varphi^{*} \pi^{*} w_{2}
$$

is in the ideal generated by $a\left[v_{1}\right]$ and $a\left[v_{3}\right]$. 
Suppose it was. Then there would be a relation of the type

$$
\begin{aligned}
& a^{3}\left[w_{1} v_{3}-(-1)^{\left|v_{1}\right|} v_{1} w_{2}\right] \\
& \quad=a\left[v_{1}\right]\left(f^{*} \xi_{1}+a \zeta_{1}^{1}+\cdots+a^{k-1} \zeta_{1}^{k-1}\right) \\
& \quad+a\left[v_{3}\right]\left(f^{*} \xi_{3}+a \zeta_{3}^{1}+\cdots+a^{k-1} \zeta_{3}^{k-1}\right) \\
& \quad=a\left(\left[v_{1}\right] i^{*} \xi_{1}\left[v_{3}\right] i^{*} \xi_{3}\right)+a^{2}\left(\left[v_{1}\right] \zeta_{1}^{1}+\left[v_{3}\right] \zeta_{3}^{1}\right) \\
& \quad+\cdots+a^{k-1}\left(\left[v_{1}\right] \zeta_{1}^{k-2}+\left[v_{3}\right] \zeta_{1}^{k-2}\right)+a^{k}\left(\left[v_{1}\right] \zeta_{1}^{k-1}+\left[v_{3}\right] \zeta_{3}^{k-1}\right) .
\end{aligned}
$$

Expanding $a^{k}$ and using again that the result is a direct sum, we look at the coefficient of $a^{3}$. Comparing both sides we see that it equals $\left[w_{1} v_{3}-(-1)^{\left|v_{1}\right|} v_{1} w_{2}\right]$, so

$$
\left[w_{1} v_{3}-(-1)^{\left|v_{1}\right|} v_{1} w_{2}\right]=\left[v_{1}\right] \zeta_{1}^{2}+\left[v_{3}\right] \zeta_{3}^{2}-c_{k-3} \zeta_{1}^{k-1}\left[v_{1}\right]+c_{k-3} \zeta_{3}^{k-1}\left[v_{3}\right] .
$$

But this contradicts the fact that $\left[w_{1} v_{3}-(-1)^{\left|v_{1}\right|} v_{1} w_{2}\right] \notin\left(\left[v_{1}\right],\left[v_{3}\right]\right)$.

\section{EXAmples}

In this section we give concrete examples where the blow-up procedure can be used to create manifolds satisfying the Lefschetz property. The examples we produce will also have nontrivial Massey products, therefore producing a counterexample to the conjecture of Babenko and Taimanov.

Our starting point is nilmanifolds, i.e., compact quotients of a nilpotent Lie group by a maximal lattice. It is a result of Benson and Gordon [3] that nontoroidal nilmanifolds never satisfy the Lefschetz property. Also, Nomizu's theorem 22 implies that the Lie algebra of the correspoding Lie group with its differential $\left(\bigwedge^{\bullet} \mathfrak{g}^{*}, d\right)$ furnishes a minimal model for the nilmanifold, therefore, no nontoroidal nilmanifold is formal. Indeed, it is a result of Cordero et al. 6] that they always have nontrivial (maybe higher order) Massey products.

The simplest nilmanifold with the properties we need is the one obtained from the product of two copies of the Heisenberg group.

Example 4.1. If $G$ is the 3-dimensional Heisenberg group, then the Lie algebra has a basis formed by the left invariant vector fields whose values at the identity are

$$
\partial_{1}=\left(\begin{array}{ccc}
0 & 1 & 0 \\
0 & 0 & 0 \\
0 & 0 & 0
\end{array}\right) ; \partial_{2}=\left(\begin{array}{ccc}
0 & 0 & 0 \\
0 & 0 & 1 \\
0 & 0 & 0
\end{array}\right) ; \partial_{3}=\left(\begin{array}{ccc}
0 & 0 & -1 \\
0 & 0 & 0 \\
0 & 0 & 0
\end{array}\right) .
$$

Then we check that $\left[\partial_{1}, \partial_{2}\right]=-\partial_{3}$ and $\left[\partial_{1}, \partial_{3}\right]=\left[\partial_{2}, \partial_{3}\right]=0$. Therefore, the quotient manifold, $\mathbb{H}$, of the 3 -Heisenberg group by the lattice generated by $\exp \partial_{i}$, will have Chevalley-Eilenberg complex $\left(\wedge^{\bullet} \mathfrak{g}^{*}, d\right)$ generated by the invariant 1 -forms $e_{i}$ dual to the $\partial_{i}$ related by

$$
d e_{1}=d e_{2}=0 ; d e_{3}=e_{1} \wedge e_{2} .
$$

So, this manifold is the nilmanifold associated to the Lie algebra with structure $(0,0,12)$.

Hence $H^{1}(\mathbb{H})$ is generated by $\left\{e_{1}, e_{2}\right\}, H^{2}$ by $\left\{e_{13}, e_{23}\right\}$ and $H^{3}$ by $\left\{e_{123}\right\}$, where, as usual, $e_{i j}$ is short for $e_{i} \wedge e_{j}$. Therefore $b_{1}=b_{2}=2$ and $b_{0}=b_{3}=1$. 
Massey products. With the relations above for $e_{1}, e_{2}$ and $e_{3}$ we get that

$$
e_{1} \wedge e_{2}=d e_{3} \text { and } e_{2} \wedge e_{1}=d\left(-e_{3}\right) \text {. }
$$

Therefore we can form the Massey product $\left\langle e_{1}, e_{2}, e_{1}\right\rangle=e_{3} \wedge e_{1}+e_{1} \wedge\left(-e_{3}\right)=$ $-2 e_{13} \neq 0$. Observe that in this case, since $e_{1} \wedge e_{2}$ is exact, Massey products have no indeterminacy and the above is a nontrivial one in $\mathbb{H}$.

Example 4.2. Now consider the product $\mathbb{H} \times \mathbb{H}=(0,0,12,0,0,45)$. The triple product $\left\langle e_{1}, e_{2}, e_{1}\right\rangle$ is still nonzero. Further, the form

$$
\omega=e_{14}+e_{23}+e_{56}
$$

is closed and has top power $6 e_{123456}$, which is everywhere nonvanishing. Hence $\omega$ is a symplectic form in $\mathbb{H} \times \mathbb{H}$.

It is easy to see that the kernel of $\omega: H^{2} \rightarrow H^{4}$ is $\operatorname{span}\left\{e_{25}\right\}$ and the kernel of $\omega^{2}$ in $H^{1}$ is $\operatorname{span}\left\{e_{2}, e_{5}\right\}$.

In $\mathbb{H}$, consider the path

$$
\alpha(t)=\exp \left(t\left(\partial_{1}+\partial_{2}+\partial_{3}\right)\right)=\left(\begin{array}{ccc}
1 & t & t+\frac{1}{2} t^{2} \\
0 & 1 & t \\
0 & 0 & 1
\end{array}\right), t \in[0,2] .
$$

Then $\alpha^{\prime}=\partial_{1}+\partial_{2}+\partial_{3}$ and, besides this, $\alpha(2) \approx \alpha(0)$, hence this is a circle.

In $\mathbb{H} \times \mathbb{H}$ there are two copies of $\alpha$ (one in each factor) making a torus $T^{2}$. A basis for the tangent space of this torus is given by $\left\{\partial_{1}+\partial_{2}+\partial_{3}, \partial_{4}+\partial_{5}+\partial_{6}\right\}$. The symplectic form evaluated on this basis equals 1 everywhere, hence this torus is a symplectic submanifold. On the other hand, $e_{25}$ evaluated on this basis also equals 1 everywhere.

Therefore, by Theorems 2.1 and 3.1 the blow-up $M^{6}$ of $\mathbb{H} \times \mathbb{H}$ along this torus satisfies the Lefschetz property (for $\varepsilon$ small enough) and has a nontrivial triple product.

Example 4.3. Still, let $M$ be the manifold from the previous example. We can change the symplectic form slightly so that it is rational and still satisfies the Lefschetz property. Hence an appropriate multiple of it will represent an integral cohomology class and hence, by Donaldson's theorem [8], it is Poincaré dual to a symplectic submanifold $\left(N^{4}, \omega\right)$. Using Fernández and Muñoz' result on formality of Donaldson submanifolds [9], $N$ still satisfies the Lefschetz property, and by Donaldson's theorem the inclusion $N \hookrightarrow M$ induces an isomorphism $H^{1}(M) \cong H^{1}(N)$ and an injection $H^{2}(M) \hookrightarrow H^{2}(N)$. Now, the Massey product in $M$ comes from three 1-forms and therefore still exists in $N$ and further, since we have an injection in $H^{2}$, this product is nonzero in $N$. So $N$ is a nonformal symplectic 4-manifold satisfying the Lefschetz property.

Example 4.4. Let $\left(N^{4}, \sigma\right)$ be the manifold obtained in Example 4.3 that has a nontrivial triple product and satisfies the Lefschetz property. By construction, $\sigma$ is an integral cohomology class, therefore, $(N, \sigma)$ can be symplectically embedded in $\mathbb{C} P^{6}$, by Gromov's Embedding Theorem [11, 25]. By Theorem 2.2, the blow-up of $\mathbb{C} P^{6}$ along $N$ will have the Lefschetz Property. According to Theorem 3.1, it will have a nonvanishing triple product (and thus is not formal) and from Theorem 1.1 it is simply connected. 


\section{ACKNOWLEDGMENTS}

I thank Oliver Thomas for comments on determinants which were useful in Lemma 2.6 and Vicente Muñoz for the argument used in Example4.3, I also thank Marisa Fernández and Marco Gualtieri for helpful suggestions and Nigel Hitchin for inspiring discussions, guidance and help with the editing of the text.

\section{REFERENCES}

1. I. K. Babenko and I. A.. Taimanov, Massey products in symplectic manifolds, Sb. Math. 191 (2000), 1107-1146. MR1786415 (2002d:53116)

2. _ On nonformal simply connected symplectic manifolds, Siberian Math. J. 41 (2000), 204-217. MR1762178 (2001g:57051)

3. C. Benson and C. S. Gordon, Kähler and symplectic structures on nilmanifolds, Topology $\mathbf{2 7}$ (1988), 513-518. MR0976592 (90b:53042)

4. J. Brylinski, A differential complex for Poisson manifolds, J. Differential Geometry 28 (1988), 93-114. MR0950556 (89m:58006)

5. G. R. Cavalcanti, New aspects of the $d d^{c}$-lemma, Ph.D. thesis, Oxford University, 2004, math.DG/0501406.

6. L. Cordero, M. Fernández, and A. Gray, Symplectic manifolds with no Kähler structure, Topology 25 (1986), 375-380. MR0842431 (87j:53051)

7. P. Deligne, P. Griffiths, J. Morgan, and D. Sullivan, Real homotopy theory of Kähler manifolds, Invent. Math. 29 (1975), 245-274. MR0382702 (52:3584)

8. S. Donaldson, Symplectic submanifolds and almost-complex geometry, J. Differential Geometry 44 (1996), 666-705. MR.1438190 (98h:53045)

9. M. Fernández and V. Muñoz, Formality of Donaldson submanifolds, Math. Z. 250 (2005), 149-175. MR2136647 (2006a:53098)

10. R. E. Gompf, A new construction of symplectic manifolds, Ann. of Math. (2) 142 (1995), 527-595. MR1356781 (96j:57025)

11. M. Gromov, A topological technique for the construction of differential equations and inequalities, Actes Congr. Internat. Math. (Nice 1970), vol. 2, 1971, pp. 221-225. MR0420697 (54:8709)

12. M. Gualtieri, Generalized complex geometry, Ph.D. thesis, Oxford University, 2003, math.DG/0401221.

13. N. Hitchin, Generalized Calabi-Yau manifolds, Quart. J. Math. Oxford 54 (2003), 281-308. MR2013140 (2004h:32024)

14. R. Ibáñez, Y. Rudyak, A. Tralle, and L. Ugarte, On certain geometric and homotopy properties of closed symplectic manifolds, Proceedings of the Pacific Institute for the Mathematical Sciences Workshop "Invariants of Three-Manifolds" (Calgary, AB, 1999), vol. 127, 2003, pp. 33-45. MR.1953319 (2003j:53139)

15. J. L. Koszul, Crochet de Schouten-Nijenhuis et cohomologie, Elie Cartan et les meth. d'aujourd'hui, Astérisque hors-série, 1985, pp. 251-271. MR0837203 (88m:17013)

16. G. Lupton and J Oprea, Symplectic manifolds and formality, J. Pure Appl. Algebra 91 (1994), 193-207. MR1255930 (95d:55010)

17. O. Mathieu, Harmonic cohomology classes of symplectic manifolds, Comment. Math. Helv. 70 (1990), 1-9. MR $1314938(96 \mathrm{e}: 58004)$

18. D. McDuff, Examples of simply-connected symplectic non-Kählerian manifolds, J. Differential Geometry 20 (1984), 267-277. MR0772133(86c:57036)

19. D. McDuff and D. Salamon, Introduction to symplectic topology, Oxford Mathematical Monographs, Oxford University Press, 1995. MR.1373431 (97b:58062)

20. S. A. Merkulov, Formality of canonical symplectic complexes and Frobenius manifolds, Internat. Math. Res. Notices 14 (1998), 727-733. MR1637093 (99j:58078)

21. T. J. Miller, On the formality of $k-1$ connected compact manifolds of dimension less than or equal to $4 k-2$, Illinois J. Math. 23 (1979), 253-258. MR0528561 (80j:55017)

22. K. Nomizu, On the cohomology of compact homogeneous spaces of nilpotent Lie groups, Ann. of Math. (2) 59 (1954), 531-538. MR0064057 (16:219c)

23. A. N. Parshin, A generalization of the Jacobian variety (Russian), Isvestia 30 (1966), 175-182. MR0196770(33:4956) 
24. Y. Rudyak and A. Tralle, On Thom spaces, Massey products and non-formal symplectic manifolds, Internat. Math. Res. Notices 10 (2000), 495-513. MR1759504 (2001h:53128)

25. D. Tischler, Closed 2-forms and an embedding theorem for manifolds, J. Differential Geometry 12 (1977), 229-235. MR0488108 (58:7677)

26. D. Yan, Hodge structure on symplectic manifolds, Adv. in Math. 120 (1996), 143-154. MR.1392276 (97e:58004)

Mathematical Institute, University of Oxford, St. Giles 24-29, Oxford, OX1 3BN, UNITED KINGDOM

E-mail address: gilrc@maths.ox.ac.uk 